\title{
Systematic and comprehensive analysis of the dose- response characteristics of a morning quality check of a linear accelerator and an important application of accelerator performance prediction
}

\author{
D. Jiang1, X. Wang1, Z. Dai ${ }^{2}$, J. Shen', D. Wang1, Z. Bao' ${ }^{1}$, C. Chen', \\ H.L. Zhao', J. Chen', C. Xie ${ }^{1}$, J. Zhang1 ${ }^{*}$, H. Liu1 ${ }^{*}$ \\ ${ }^{1}$ Department of Radiation and Medical Oncology, Hubei Key Laboratory of Tumor Biological Behaviors, Hubei \\ Cancer Clinical Study Center, Zhongnan Hospital of Wuhan University, Wuhan, China \\ ${ }^{2}$ Cancer hospital Chinese Academy of Medical Sciences, Shenzhen Center, Shenzhen, China
}

\section{- Original article}

\section{*Corresponding authors:}

Hui Liu and Jun Zhang, Ph.D., E-mail:

hbzkznyy@163.com

zhangjun110@whu.edu.cn

Revised: April 2020

Accepted: May 2020

Int. J. Radiat. Res., October 2020; 18(4): 841-851

DOI: 10.18869 /acadpub.ijrr.18.4.841

\begin{abstract}
Background: This paper aimed to analyze the output constancy of a medical linear accelerator using PTW QUICKCHECK ${ }^{\text {webline }}$ and studied the sensitivity of the PTW QUICKCHECK ${ }^{\text {webline }}$. Materials and Methods: The paper statistically analyzed the output doses of $6 \mathrm{MV}$ and $10 \mathrm{MV}$ of photons and $6 \mathrm{MeV}, 9 \mathrm{MeV}$, and $12 \mathrm{MeV}$ of electrons from a medical linear accelerator measured before the daily treatment to assess the output stability of a medical linear accelerator. Some modifications were introduced by artificially altering the external irradiation conditions, and the percent variations from baseline values were noted. The gantry angle was changed and some deviations were established in the vertical, longitudinal and lateral directions to study the

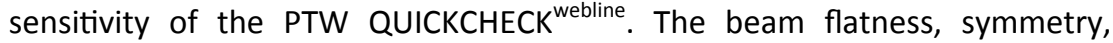
radiation quality and output energy of $6 \mathrm{MV}$ of photon energy were statistically analyzed. Results: Among the measurements, no parameters exceeded the tolerance of $\pm 3 \%$. QUICKCHECK ${ }^{\text {webline }}$ was capable of detecting the variations in the central axis dose, flatness, symmetry and radiation quality under the testing conditions. Similar to the photon energy, electron energy measurements also confirmed that the detector was sensitive to a small variation in output introduced by the testing conditions. An important application of accelerator performance prediction in this study confirms the irreplaceable and important function of morning quality checks of a linear accelerator. Conclusions: The output dose measured before daily treatment using PTW QUICKCHECK ${ }^{\text {webline }}$ to analyze the linear accelerator output constancy helps to decrease the system error, effectively reduces the errors of the accelerator system, and avoids serious mistakes.
\end{abstract}

Keywords: PTW QUICKCHECK webline, constancy, morning check, radiation quality, routine quality assurance.

\section{INTRODUCTION}

Quality assurance $(\mathrm{QA})$ is the process of verifying whether a product or machine function is within the range of some criteria. The QA of a linear accelerator plays an important role in precise tumor radiotherapy $(1,2)$. Linac $Q A$ is designed to ensure that the device does not significantly deviate from its baseline values. The purpose of daily, monthly and annual QA was established according to the American Association of Physicists in Medicine TG40 and TG142 ${ }^{(3,4)}$. Some scholars have concluded that a deviation ranging from 7 to $10 \%$ of dose delivery results in clinically detectable effects on tumor and normal tissues by studying the tumor 
control probability (TCP) and normal tissue complication probability (NTCP) (5-10). According to retrospective studies, the clinical tolerance for random and systematic uncertainties and variations in the output trends of a linear accelerator should be within $\pm 3 \%$ (11-17). Some scholars suggest that the detection equipment requires sufficient accuracy and precision to detect this level of variation $(18,19)$.

Since the absolute dose delivered to the patient is an important factor in determining the outcome of treatment, linear accelerator output constancy has always been an important part of a regular QA procedure. Some quick check devices, such as diode and ionization chamber-based array detectors, are becoming increasingly popular in the quality control of linear accelerator parameters (19). Although QUICKCHECKwebline (PTW, Freiburg, Germany) has been used as a daily QA tool in many radiotherapy centers, few scholars have published studies verifying the performance of this device for assessing the constancy of a medical linear accelerator (20,21). The purpose of this study was to assess the performance of QUICKCHECKwebline.

The QUICKCHECKwebline device consists of ionization chambers with inherent build up designed for a routine constancy assessment of linear accelerator beam parameters, such as the dose output, flatness, symmetry, radiation quality and irradiation time (radiation and light field size checks). The device also contains software features that create a baseline template and a record of routine data (after an analysis monitoring the performance of linear accelerators). Tedious installation procedures and long training times are not required. QUICKCHECKwebline is a cable-free, truly wireless system, with all required essential components built in for an easy and convenient operation. After an initial set-up, it is ready for daily use. The purpose of this report is to assess the performance of the QUICKCHECKwebline device as a daily quality assurance tool and derive a set of recommendations for its use. To our knowledge, this study is the first to study PTW QUICKCHECKwebline in detail. The results of sensitivity analyses conducted in various directions are included in this article.

\section{MATERIALS AND METHODS}

All measurements were conducted using Varian iX (Sn: 6324) linear accelerators at Wuhan University Zhongnan Hospital with photon energies of 6 and $10 \mathrm{MV}$ and electron energies of 6,9 and $12 \mathrm{MeV}$. The accelerators operate at a dose rate of $400 \mathrm{MU} / \mathrm{min}$ for electrons. After comparing the measurement data on dose output, symmetry, flatness and index for radiation quality (BQF) of PTW QUICKCHECKwebline with the ionization chamber, PTW QUICKCHECKwebline is used as the measuring instrument for morning quality checks of the linear accelerator.

\section{QUICKCHECK ${ }^{\text {webline }}$ device}

The baseline measurements of QUICKCHECKwebline were performed with a field size $10 \times 10$ and $20 \times 20 \mathrm{~cm}^{2}$ at a 100 SSD for 100 MU. QUICKCHECKwebline has 13 detectors, which are used to measure the dose, dose rate, and beam delivery time and to calculate flatness, symmetry, and index for BQF. The baseline template should be recalibrated if a drift in Linac output is observed or if an error occurred in the initial linear accelerator output calibration.

\section{Short-term reproducibility and linearity}

The reproducibility and linearity of the device were first assessed to ensure the capability of QUICKCHECKwebline to detect small variations in output. Linearity was tested by delivering set Monitor Units (MU) to the QUICKCHECKwebline device with no additional build-up-in the interval between 80 and $120 \mathrm{MU}$ at $5 \mathrm{MU}$ increments and a standard $10 \times 10 \mathrm{~cm}^{2}$ at $100 \mathrm{~cm} \mathrm{SSD}$ and $6 \mathrm{MV}$ of energy. The data were then compared to a corresponding linearity test performed using a Farmer ionization chamber. The values measured using both systems were normalized to the measured value of $100 \mathrm{MU}$ to directly show the deviation of QUICKCHECK webline and the Famer chamber from linearity. Long- and shortterm reproducibility was tested for a set number of MUs using both QUICKCHECK ${ }^{\text {webline }}$ and the

Int. J. Radiat. Res., Vol. 18 No. 4, October 2020 
Farmer ionization chamber and the percent standard deviation was derived from these measurements. In this study, the author will set the error to study the sensitivity of the detector. For example, moving bed values in $\mathrm{X}, \mathrm{Y}$, and $\mathrm{Z}$ directions were used to detect the sensitivity of the detector response.

\section{Long-term reproducibility and output constancy}

Output measurements recorded using a PTW Farmer Chamber were compared to the baseline values, and the percent error was plotted against the corresponding percent error of QUICKCHECKwebline. The central axis chamber (CAX) on the QUICKCHECKwebline device is primarily used to measure the output of the required beam. The temperature-pressurecorrected measurements (QUICKCHECKwebline and Farmer ionization chamber) recorded using both detectors were compared to the corresponding baseline value.

\section{Calculations}

\section{Air density corrections}

The QUICKCHECK measuring chambers are vented and require air density correction. Notably, QUICKCHECK will automatically perform the air density correction. The correction factor $\mathrm{K}_{\mathrm{TP}}$ for air density correction is calculated using equation 1 :

$K_{T P}=\frac{(273.2+T) * P_{0}}{\left(273.2+T_{0}\right) * P}$

Where

$\mathrm{T}$ is the temperature in $\left({ }^{\circ} \mathrm{C}\right)$ measured by QUICKCHECK.

$\mathrm{P}$ is the atmospheric pressure in $(\mathrm{hPa})$ measured by QUICKCHECK.

$\mathrm{T}_{0}$ is the temperature for calibration $20^{\circ} \mathrm{C}$.

$\mathrm{P}_{0}$ is the atmospheric pressure for calibration $1013.25 \mathrm{hPa}$.

\section{Dose values}

The dose values Di for all measuring chambers are calculated using equation 2 :

$D_{i}=M_{i} \times N_{i} \times K_{T P}$
Where

$\mathrm{M}_{\mathrm{i}}$ is the measured charge of measuring chamber $\mathrm{i}$.

$\mathrm{N}_{\mathrm{i}}$ is the ${ }^{60}$ Co calibration factor of measuring chamber $\mathrm{i}$.

$\mathrm{K}_{T P}$ is the correction factor for air density correction (refer to equation (1))

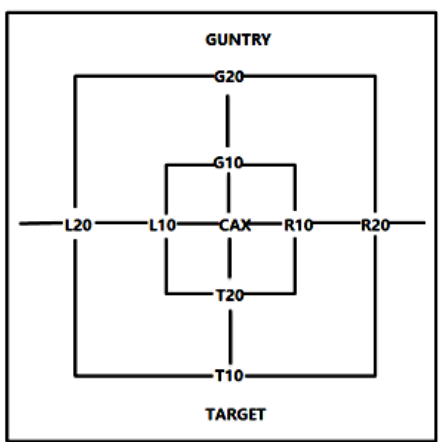

Figure 1. The QUICKCHECK measuring chambers for dose measurements.

\section{Normalization factor $\boldsymbol{k}_{\text {norm }}$}

QUICKCHECK allows the user to normalize the evaluation values with the normalization factor $\mathrm{k}_{\text {norm. }}$. The evaluation values of the current measurement and all subsequent measurements will then be multiplied by this normalization factor. QUICKCHECK will automatically calculate a normalization factor with the Normalize function.

\section{Calculating evaluation values Central axis dose CAX}

Equation 3 was used to calculate central axis dose as follows:

$$
\mathrm{CAX}=\left(\mathrm{k}_{\mathrm{norm}}\right)_{\mathrm{CAX}} \times \mathrm{D}_{\text {cax }} .
$$

Where

$\left(\mathrm{k}_{\mathrm{norm}}\right)_{\mathrm{CAX}}$ is the normalization factor for the central axis dose.

$D_{\text {cax }}$ is the central chamber dose calculated according to equation (2).

\section{Flatness of the field}

The central chamber and the following ionization chambers are used to calculate the flatness:

-field of $10 \mathrm{~cm}$ x $10 \mathrm{~cm}$ : ionization chambers 
CAX, T10, L10, G10 and R10

-field of $20 \mathrm{~cm} \times 20 \mathrm{~cm}$ : ionization chambers CAX, T20, T10, L20, L10, G20, G10, R20 and R10.

From the measured values of these ionization chambers, the maximum dose value $\mathrm{D}_{\max }$ and the minimum dose values $\mathrm{D}_{\text {min }}$ are determined.

The calculation of the flatness depends on the modality and on the evaluation protocol. The protocol to use is set when the worklists are created in the Worklist Generator software. The algorithm selected is as described below.

\section{Evaluation algorithm}

This algorithm assesses the quality of the flatness normalized to $100 \%$ according to equation 4:

Flatness $=100 *\left(k_{\text {norm }}\right)_{\text {Flat }} * \frac{D_{\max }}{D_{\min }}$

$\left(\mathrm{k}_{\text {norm }}\right)_{\text {Flat }}$ is the normalization factor for flatness

$D_{\max }$ is the maximum dose value of the 5 or 9 ionization chambers

$\mathrm{D}_{\text {min }}$ is the minimum dose value of the 5 or 9 ionization chambers

\section{Symmetry}

Symmetry is analyzed separately for the gun-target direction and left-right direction. The calculation of the symmetry $S$ depends on the modality and on the evaluation protocol. The protocol to use is set when the worklists are in the Worklist Generator software. The algorithm selected is as follows:

Evaluation algorithm: This algorithm assesses the quality of the symmetry normalized to $100 \%$ according to equations 5 and 6 .

$$
\begin{gathered}
S_{L R}=100 *\left(k_{\text {norm }}\right)_{S y m L R} * \underset{M A X}{\operatorname{MAX}} \\
X=L 10
\end{gathered} *\left[\frac{\operatorname{MAX}\left(D_{-X}, D_{X}\right)}{\operatorname{MIN}\left(D_{-X}, D_{X}\right)}\right]
$$

Where

$\left(\mathrm{k}_{\text {norm }}\right)_{\text {symLR }}$ is the normalization factor for symmetry in the left-right direction

$\left(\mathrm{k}_{\text {norm }}\right)_{\text {symGT }}$ is the normalization factor for symmetry in the gun-target direction
$D_{-}, D_{x}$ is dose values for the ionization chambers at the chamber positions $\mathrm{x}$ or $-\mathrm{x}$. the chamber positions $\mathrm{x}$ and $-\mathrm{x}$ are symmetrical to the central beam. (Examples: if $\mathrm{x}=\mathrm{L} 10$ then $\mathrm{x}=\mathrm{R} 10$, if $\mathrm{x}=\mathrm{G} 20$ then $-\mathrm{x}=\mathrm{T} 20$ )

$D_{\text {cax }}$ is the central chamber dose calculated according to equation (2).

\section{Index for radiation quality $B Q F$}

The index for the radiation quality $\mathrm{BQF}$ can only be determined for the following field sizes:

Photons: field size $10 \mathrm{~cm} * 10 \mathrm{~cm}$

Electrons: field size $20 \mathrm{~cm}^{*} 20 \mathrm{~cm}$

When determining the index for the radiation quality $\mathrm{BQF}$, build-up plates must not be used. An open field must be used to determine the index for the radiation quality $\mathrm{BQF}$. The central chamber and one of the four ionization chambers for radiation quality are used to calculate the index for the radiation quality BQF. The index for the radiation quality $\mathrm{BQF}$ is calculated using the equation 7:

$\mathrm{BQF}=\left(k_{\text {norm }}\right)_{B Q F} *$ Polynom $\left(\frac{D_{E i}}{D_{C A X}}\right)$

Where

$\left(\mathrm{k}_{\mathrm{norm}}\right)_{\mathrm{BQF}}$ is the normalization factor for the index for the radiation quality

$D_{E i}$ is the dose of the corresponding ionization chamber for radiation quality

$D_{\text {cax }}$ is the central chamber dose calculated according to formula (2).

\section{Statistical analysis}

Statistical analyses were performed using SPSS® Statistics 19.0 software (IBM Corp., New York, NY; formerly SPSS Inc., Chicago, IL). If $\mathrm{p}$-values $<0.05$, the differences were considered statistically significant.

\section{RESULTS}

\section{Short-term reproducibility and linearity}

First, the short-term reproducibility of QUICKCHECK ${ }^{\text {webline }}$ was tested by using a set of MUs ranging from 80 to $120 \mathrm{MU}$ at $5 \mathrm{MU}$ increments and $6 \mathrm{MV}$ of energy using $10 \times 10$

Int. J. Radiat. Res., Vol. 18 No. 4, October 2020 
$\mathrm{cm}^{2}$ field size at $100 \mathrm{~cm}$ SSD. As shown in figure 2, QUICKCHECKwebline readings were linear for all measured energies with set monitor units (80-120 MU) compared with the Farmer chamber. The two curves of QUICKCHECKwebline output and PTW chamber output are almost parallel.

\section{Long-term reproducibility and output constancy}

Measurements recorded by QUICKCHECKwebline and the PTW Farmer ionization chamber were compared weekly over a 6-month period to assess the long-term reproducibility. As shown in figure 3, the QUICKCHECKwebline device produced reproducible and consistent results during this experiment for up to 6 months. Output variations observed between QUICKCHECK ${ }^{\text {webline }}$ and the Farmer chamber were within $1 \%$ at $6 \mathrm{MV}$ and $10 \mathrm{MV}$ in the 6 month period, as shown in figure 3 .

After the analysis of the long-term reproducibility and output constancy, a morning quality check was performed daily before treatment for a month. The output values of the QUICKCHECKwebline are shown in figure 4. It can be seen that the accelerator output fluctuated in a very small range $(<+1 \%)$ near the standard value and that its performance remained relatively stable.

\section{Sensitivity of the PTW QUICKCHECK webline detector}

Some modifications were introduced by artificially altering the external irradiation conditions, and the percent variations from baselines were noted. The gantry angle was changed, and some deviations in the vertical, longitudinal (gun-target direction) and lateral (left-right direction) directions were set in the next experiment. Then, the beam flatness, symmetry, radiation quality $\mathrm{BQF}$ and output energy of $6 \mathrm{MV}$ of photon energy were statistically analyzed.

\section{In the vertical direction}

The output of PTW QUICKCHECKwebline was studied in the vertical direction. The deviation was a $1 \mathrm{~mm}$ increment, and the results are shown in figure 6.

The abscissa value represents the positive value of the raised bed and the negative value of the lowered bed. The FLAT, CAX and BQF values were more sensitive in the vertical direction. The change in this direction exerted little effect on SYMGT and SYMLR. Notably, the absolute dose decreased as the SSD increased, but the flatness showed the opposite trend. The flatness increased as the SSD increased, and then reached $100 \%$.

\section{In the left-right direction}

In the left-right direction, the deviation was a $1 \mathrm{~mm}$ increment, and the results are shown in figure 7.

\section{In the gun-target direction}

In the gun-target direction, the deviation was a $1 \mathrm{~mm}$ interval, and the results are shown in figure 8.

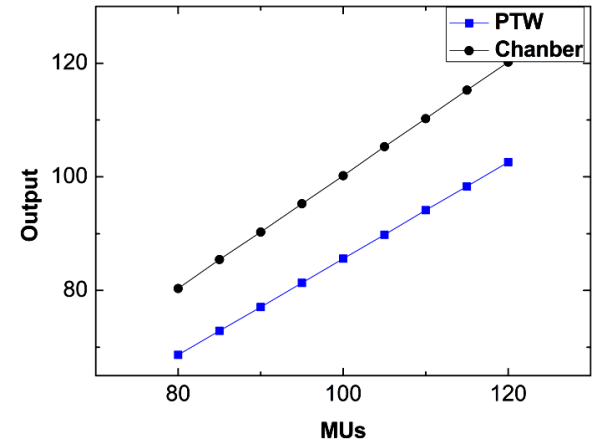

Figure 2. Linearity of the measurements recorded by QUICKCHECKwebline and the Farmer ionization chamber.
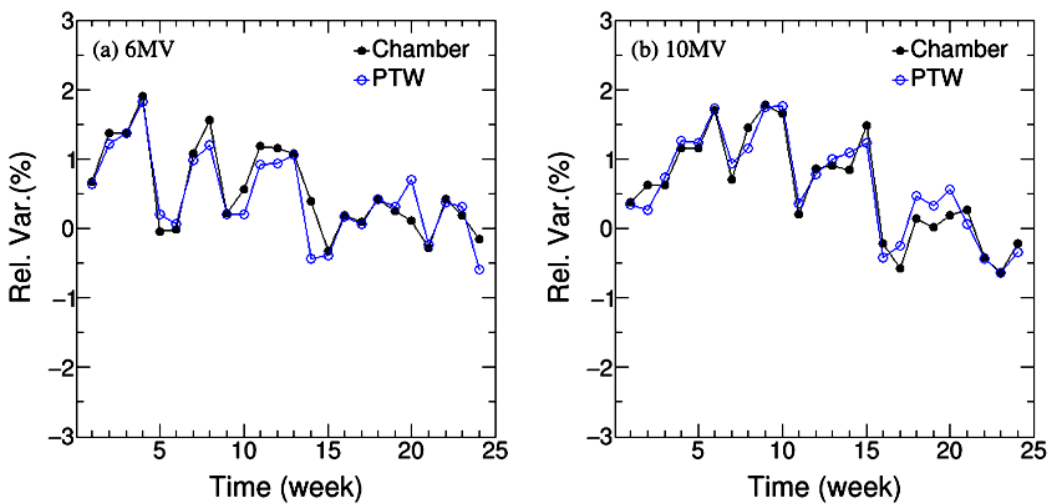

Figure 3. Variations in output constancy from baseline values for QUICKCHECK ${ }^{\text {webline }}$ and the Farmer ionization chamber measuring a Varian iX (Sn: 6324) linear accelerator at an energy of (A) $6 \mathrm{MV}$ and (B) $9 \mathrm{MV}$. 

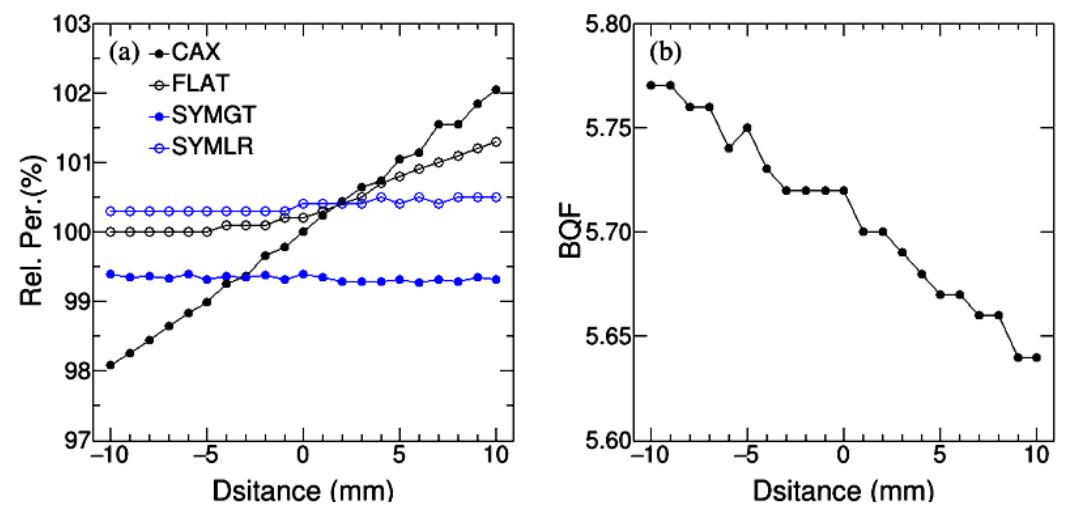

Figure 5. The output of PTW QUICKCHECKwebline in the vertical direction.
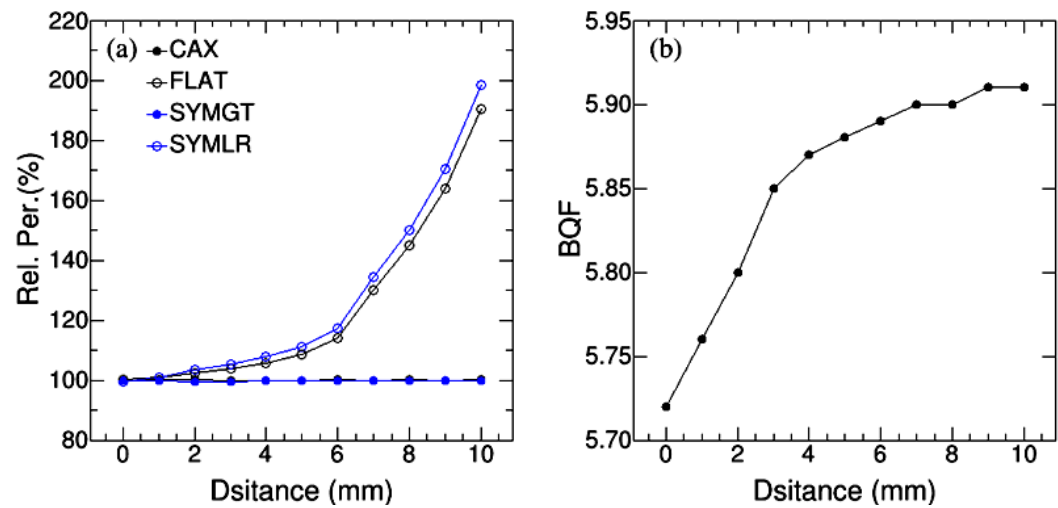

Figure 6. The output of PTW QUICKCHECKwebline in the left-right direction. The abscissa represents the distance from the center. The FLAT, SYMLR and BQF were more sensitive in the left-right direction. The change in this direction exerted little effect on SYMGT and CAX within $1 \mathrm{~cm}$.
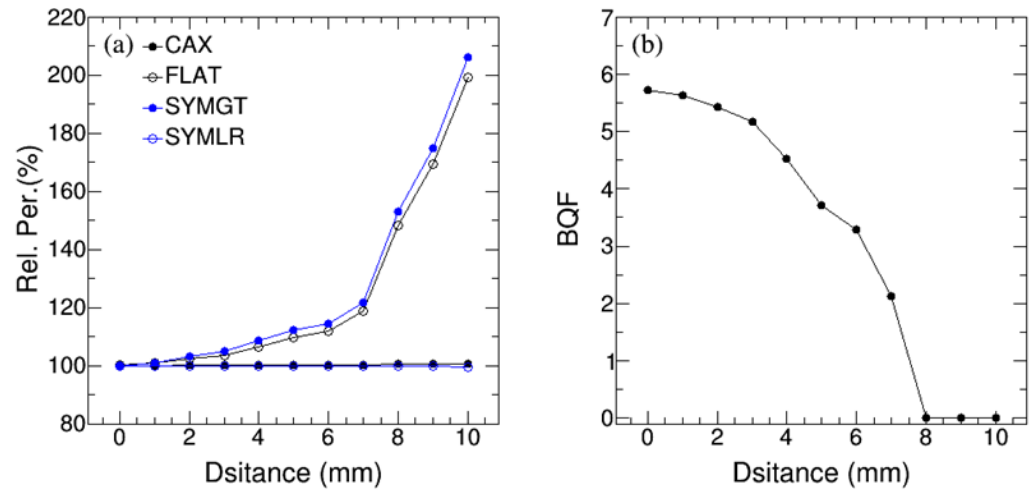

Figure 7. The output of PTW QUICKCHECKwebline in the gun-target direction.

Similar to the left-right direction, the FLAT, SYMGT and BQF were more sensitive in the gun-target direction. The change in this direction exerted little effect on SYMLR and CAX within $1 \mathrm{~cm}$. However, the trend for BQF was different. In the left-right direction, $\mathrm{BQF}$ increased with the distance from the center but decreased in the gun-target direction.

\section{Effect of the gantry angle}

The effect of gantry angle was analyzed in the present study. The gantry angle was changed in 1 degree increments, and the results are shown in figure 8.

Int. J. Radiat. Res., Vol. 18 No. 4, October 2020 

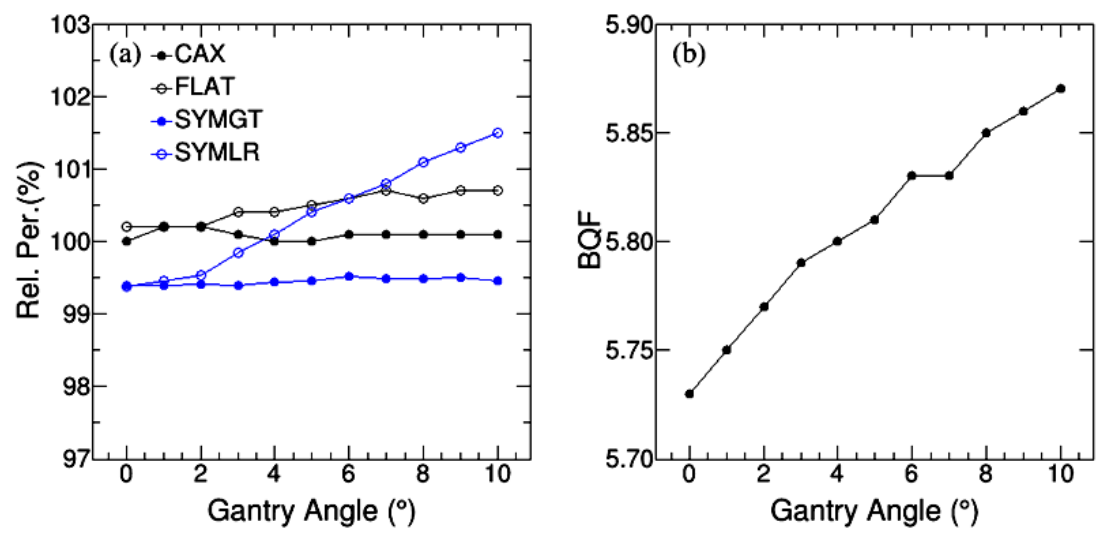

Figure 8. The effect of the gantry angle.

SYMLR and BQF increased in a small range as the gantry angle changed, whereas CAX, FLAT and SYMGT remained almost unchanged.

\section{Effect of the collimator angle}

The CAX, FLAT, SYMLR, SYMGT and BQF changed as the collimator angle changed, as shown in figure 9 .

As shown in figure 9, changing the collimator angle only affected BQF. In the case of photons, the measurements were also sensitive to depth, distance, gantry angle and collimator angle. QUICKCHECKwebline was capable of detecting the variations in the central axis dose, flatness, symmetry and radiation quality under the testing conditions. Similar to the photon energy, electron energy measurements also confirmed that the detector was sensitive to a small variation in output introduced by the testing conditions.

\section{An important application of the accelerator performance prediction}

From February 2018 to July 2018, the output
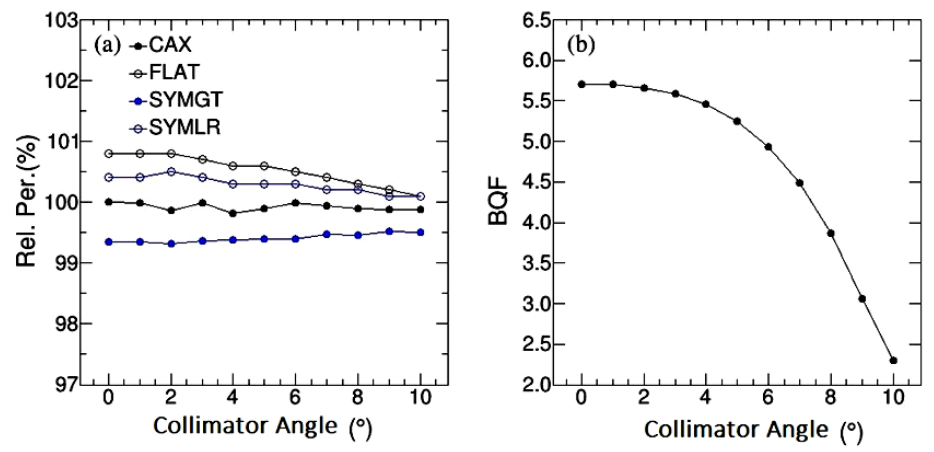

Figure 9. The effect of the collimator angle. of the accelerator was measured using the QUICKCHECKwebline as part of the morning quality check of the linear accelerator daily before the patient underwent treatment. The output of the machine was 100 MU per treatment. As shown in Figure 10, before replacing the monitoring chamber, the output deviation was within 2 percentage points. When the deviation exceeds $2 \%$, the physicist should measure and calibrate the accelerator through the ionization chamber in a timely manner to return it to the standard value. After performing a retrospective analysis of the output dose of the QUICKCHECKwebline for a period of time, the author observed consistent increases in the output values that continued to increase when the physicist lowered the output. Although the deviation met the clinical requirements, the author still suspected that the accelerator had some potential safety issue. After strict testing by physicists and factory engineers, the monitoring chamber function had failed. 

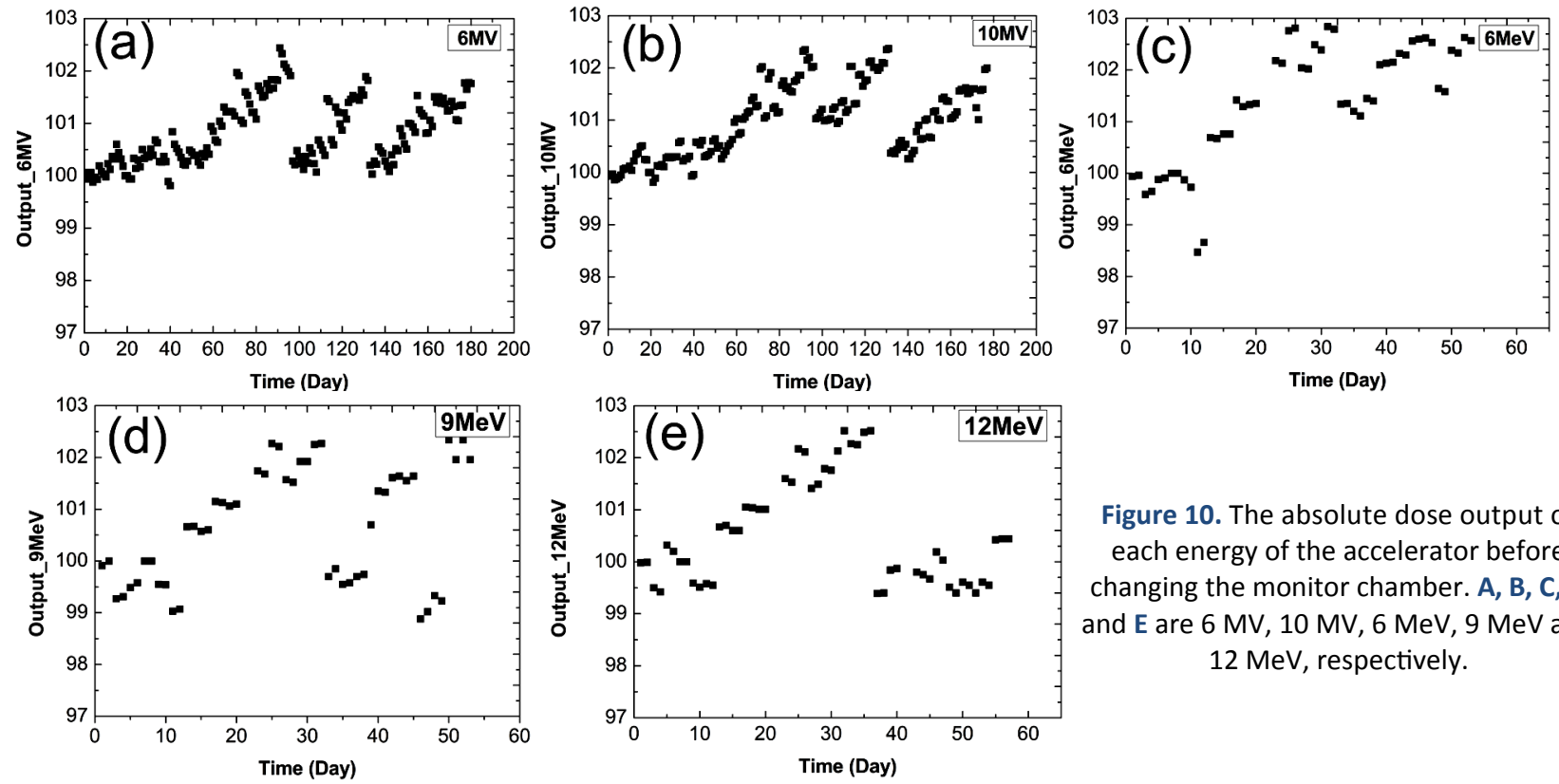

Figure 10. The absolute dose output of each energy of the accelerator before changing the monitor chamber. A, B, C, D and $\mathrm{E}$ are $6 \mathrm{MV}, 10 \mathrm{MV}, 6 \mathrm{MeV}, 9 \mathrm{MeV}$ and $12 \mathrm{MeV}$, respectively.

After replacing the monitoring chamber, the QUICKCHECKwebline output values were measured for a period of time, as shown in figure 11. The output deviation of the
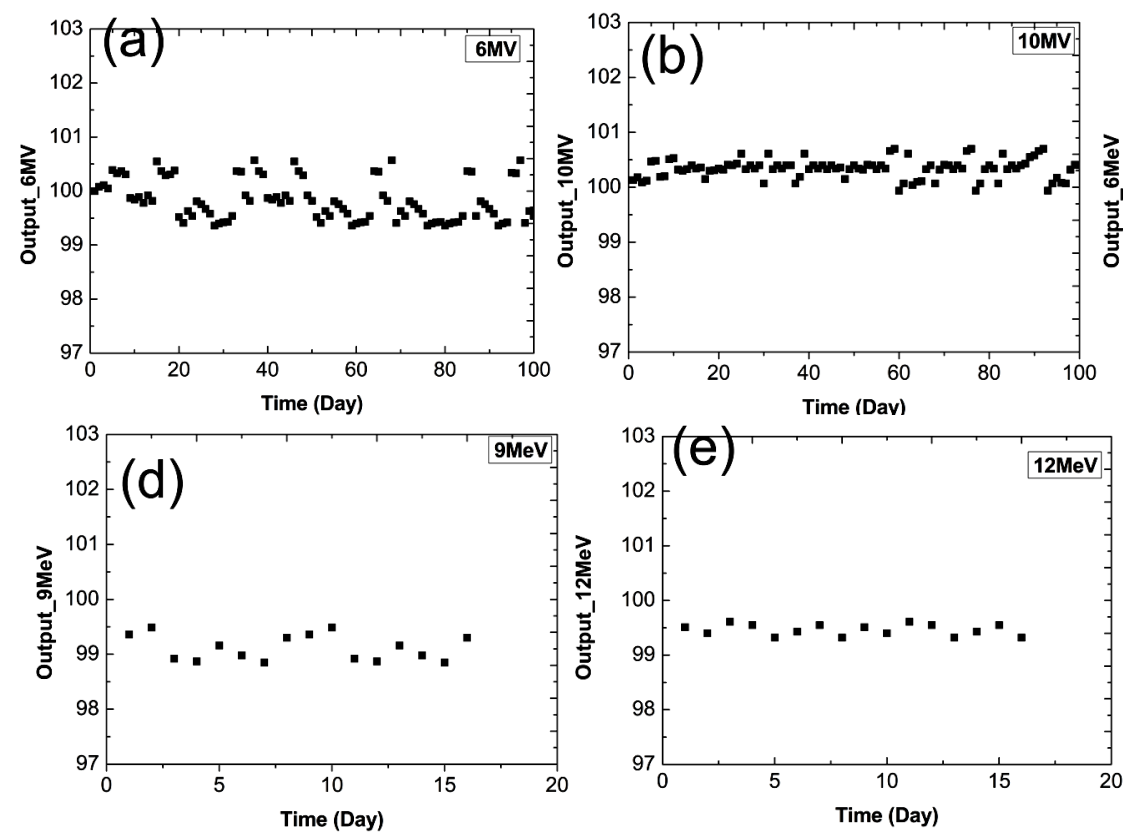

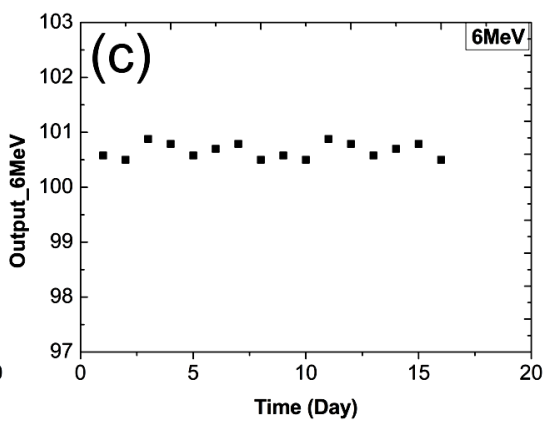

Figure 11. The absolute dose output of each energy of the accelerator before changing the monitor chamber. A, B, C, D and $\mathrm{E}$ are $6 \mathrm{MV}, 10 \mathrm{MV}, 6 \mathrm{MeV}, 9 \mathrm{MeV}$ and $12 \mathrm{MeV}$, respectively.

\section{DISCUSSION}

The fundamental purpose of radiation therapy is to administer the highest possible radiation dose to the tumor area as high and lowest possible dose to the normal organs in order to increase the TCP and reduce the NTCP. The prerequisite for achieving this goal is to ensure that the machine exhibits high stability. Various national organizations, including the 
Institute of Physics and Engineering in Medicine (IPEM), International Commission on Radiation Units, Measurements (ICRU) and American Association of Physicists in Medicine (AAPM), recommend a clinical tolerance for linear accelerator output variation of $\pm 3 \%$ to achieve the planned tumor response (4-10). This guideline also enables each radiotherapy center to appropriately reduce the monitoring frequency according to the business characteristics and workload of the particular unit. We obtained the same conclusion as Mcdermott et al. (18) We all believe that a morning quality check of the linear accelerator is useful for monitoring the stability of the output parameters of the linear accelerator at two measurements based on the ionization chamber, which can be confirmed and debugged at the ionization chamber in a timely manner when a problem is detected.

In the present study, QUICKCHECK was used to monitor the output of the linear accelerator before the daily treatment of patients with cancer. The data obtained from $6 \mathrm{MV}$ and $10 \mathrm{MV}$ $\mathrm{X}$ rays and $6 \mathrm{MeV}, 9 \mathrm{MeV}$, and $12 \mathrm{MeV}$ electron beam rays during the daily morning examination were within 3\%. As shown in figures $3 \mathrm{~A}$ and $3 \mathrm{~B}$, when the output of the morning detector is greater than $2.0 \%$, the physicist uses the ionization chamber to calibrate the absolute dose of the accelerator output in time, consistent with the findings reported by Binny et al. (19). We also obtained the variations from the Farmer chamber and compared them with the QUICKCHECKwebline, which were within $\pm 2 \%$. As shown in figure 4, the output dose of the accelerator fluctuates within the minimum range near the standard value, and its performance maintains a high stability. Thwaites et al. (6) recommended that the output deviation should be less than $3 \%$, but we should not be confident that the performance of the machine is good when the deviation is within $3 \%$. Physicists must carefully test other parameters, including the flatness, symmetry, ray quality, etc., when the output of the morning detector is consistently greater than $2.0 \%$.

A medical linear accelerator is the main equipment used in tumor radiotherapy, and its stability is an important aspect to ensure the treatment effect on patients. Physicists not only must regularly calibrate the accelerator output dose but also monitor the stability of the output dose during each dose calibration interval. Therefore, a daily output dose monitoring mechanism must be established, and an effective daily/weekly/monthly measurement system has become an essential part of accelerator system quality assurance $(3,4,22)$.

By analyzing the sensitivity of the QUICKCHECKwebline detector, changes in any parameter (including the vertical direction, gun-target direction, left-right direction, gantry angle and collimator angle) will affect the output of the machine, as shown in figures 6-9. Based on these findings, we concluded that QUICKCHECKwebline is capable of detecting modifications in the testing conditions by reporting relative changes from baseline data.

Because of its good linearity and reproducibility, the PTW QUICKCHECKwebline is an appropriate device for monitoring the constancy of the linear accelerator output. We have discussed the factors that affect the measurement capability, such as gantry angle calibration, ionization chamber measurement techniques and specifications. The department has achieved satisfactory results in daily morning QA with the PTW QUICKCHECKwebline for a Varian iX (Sn: 6324) accelerator.

We performed a retrospective analysis of a case found by QUICKCHECKwebline during morning QA, as shown in figure 10. The output of the Linac is within $3 \%$ of variation, with a consistent positive drift, which is similar to the results reported by Chan et al. (23), Grattan et al. (13) and Hossain (14). Chan et al reported a consistent positive drift for all seven energies ( $\sim 0.25 \%$ per month), Hounsell et al. reported an average monthly increase of $0.26 \% \pm 0.009 \%$ over the course of the first 4 years of use, and Hossain (14) reported an increase in the output of 3 Linacs with 9 beams by approximately $2 \%$ $4 \%$ per year over a period of more than three years, if the adjustments are artificially removed by physicists once every $3-6$ months. The deviations in our study are much larger than those values. After our careful testing, the monitoring chamber of the accelerator failed. 
However, the author is unable to clearly determine whether a necessary connection exists between the phenomenon of increasing deviation and the failure of the monitoring chamber. This phenomenon has provided some insights for the author; although the deviation of machine output is less than $3 \%$, physicists should not be confident that the machine exhibits good performance. Physicists must carefully monitor the fluctuations in the output value or the continuous changes in the output dose of the machine in one direction over a period of time. Currently, the potential safety issue of accelerator is important, and thus, the machine must be maintained and analyzed in a timely manner.

\section{CONCLUSION}

Through this study, we have verified the suitability of the PTW QUICKCHECKwebline device for routine quality assurance of the medical linear accelerator output, energy, flatness and symmetry. The PTW QUICKCHECKwebline device exhibits good linearity and reproducibility. It produces similar measurements to Farmer and Markus ionization chambers.

\section{ACKNOWLEDGEMENTS}

The authors thank the Department of Radiation and Medical Oncology, Zhongnan Hospital of Wuhan University. Thanks for all colleagues' help and guidance. This project is financially supported by Zhongnan Hospital of Wuhan University Science, Technology and Innovation Seed Fund, Project znpy2019022 and Project znpy2019095.

\section{Conflicts of interest: Declared none.}

\section{REFERENCES}

1. Saiquan $L U$, Jiazhou $W$, Jiayuan $P$, Jiang $X$, Weigang $H U$, et al. (2018) Experience of linear accelerator quality assur- ance based on the Radiation Therapy Oncology Group specification. China Oncology, 28(4): 308-31.

2. O'Daniel JC and Yin FF (2017) Quantitative approach to failure mode and effect analysis for linear accelerator quality assurance. International Journal of Radiation Oncology Biology Physics, 98(1): 56.

3. Klein EE, Hanley J, Bayouth J, Yin FF, Simon W, Dresser S, et al. (2009) Task Group 142, American Association of Physicists in Medicine. Task Group 142 report: quality assurance of medical accelerators. Med Phys, 36(9): 4197212.

4. Kutcher GJ, Coia L, Gillin M, Hanson WF, Leibel S, Morton RJ, et al. (1994) Comprehensive QA for radiation oncology: Report of AAPM Radiation Therapy Committee Task Group 40. Medical Physics, 21(4): 581.

5. Dutreix A (1984) When and how can we improve precision in radiotherapy? Radiotherapy \& Oncology, 2(4): 275-292.

6. Thwaites DJ, Mijnheer BJ, \& Mills JA (2011) Quality assurance of external beam radiotherapy. Int J Radiat Oncol Biol Phys, 39(2): 1011-1018(8).

7. Mayles W, Lake R, McKenzie A, Macaulay E, Morgan H, Jordan T, et al. (1999) Physics aspects of quality control in radiotherapy. Institute of Physics and Engineering in Medicine (IPEM) IPEM Report 811999.

8. Millar M, Cramb J, Das R, Ackerly T, Brown G \& Webb D, et al. (1997) Recommendations for the safe use of external beams and sealed brachytherapy sources in radiation oncology. Australasian Physical and Engineering Sciences in Medicine, 20: 1-35.

9. "International Commission on Radiation Units and Measurements Report 24, Determination of Absorbed Dose in a Patient Irradiated by Beams of X or Gamma Rays in Radiotherapy Procedures." Icru Report (1976).

10. Mijnheer BJ, Battermann JJ, \& Wambersie A (1987) What degree of accuracy is required and can be achieved in photon and neutron therapy? Radiotherapy \& Oncology, 8 (3): 237-252.

11. Panagiotis T and Hawkins DM (2005) Statistical process control for radiotherapy quality assurance. Medical Physics, 32(9): 2777-2786.

12. Sanghangthum T, Suriyapee $S$, Srisatit $S$, \& Pawlicki $T$ (2013) Retrospective analysis of linear accelerator output constancy checks using process control techniques. Journal of Applied Clinical Medical Physics, 14(1): 147-160.

13. Grattan MW and Hounsell AR (2011) Analysis of output trends from varian $2100 \mathrm{c} / \mathrm{d}$ and $600 \mathrm{c} / \mathrm{d}$ accelerators. Physics in Medicine \& Biology, 56(1): 11-9.

14. Hossain M (2014) Output trends, characteristics, and measurements of three mega-voltage radiotherapy linear accelerators. Journal of Applied Clinical Medical Physics, 15(4): 4783.

15. Murry R (2014) A review of the QA history for five Varian linear accelerators. J Med Imaging Radiat Oncol, 58: 289.

16. Blanck O, Masi L, Chan MK, Adamczyk S, Albrecht C, Damme MC, et al. (2016) High resolution ion chamber array delivery quality assurance for robotic radiosurgery: commissioning and validation. Phys Med, 32: 838-46.

Int. J. Radiat. Res., Vol. 18 No. 4, October 2020 
17. Sjölin M and Edmund JM (2016) Incorrect dosimetric leaf separation in IMRT and VMAT treatment planning: clinical impact and correlation with pretreatment quality assurance. Physica Medica, 32(7): 918-925.

18. Mcdermott GM and Buckle AH (2012) Monitoring linear accelerator output constancy using the ptw linacheck. Medical Dosimetry, 36(1): 71-74.

19. Binny D, Lancaster CM, Kairn T, Trapp JV, Crowe SB (2016) Monitoring daily qa 3 constancy for routine quality assurance on linear accelerators. Physica Medica, 32(11): 14791487.

20. Senz S, Walke M, Gademann G (2010) Using the PTW Quickcheck(webline) to constancy test and quality proof of medical linear accelerators[C]// Meeting of the German
-Society-Of-Radio-Oncology, 99-99.

21. Mosleh-Shirazi MA, Rahimi S, \& Karbasi S (2015) Mediumterm stability of the photon beam energy of an Elekta Compact TM linear accelerator based on daily measurements of beam quality factor. Iranian Journal of Medical Physics, 12(4): 230-234.

22. Broggi S, Cattaneo GM, Molinelli S, Maggiulli E, Del VA, Longobardi B, et al. (2008) Results of a two-year quality control program for a helical tomotherapy unit. Radiother Oncol, 86(2): 231-41.

23. Chan MF, Li Q, Tang X., Li X, Li J, Tang G, et al. (2015) Visual Analysis of the Daily QA Results of Photon and Electron Beams of a Trilogy Linac over a Five-year Period. Int J Med Phys Clin Eng Radiat Oncol, 4(4): 290-299. 
